\title{
Intervenciones para mejorar la salud psicológica y los procesos cognitivos, emocionales y comportamentales en niños y adolescentes víctimas de conflicto armado: Una revisión sistemática
}

\author{
Cristian Villanueva-Bonilla ${ }^{1,2,3}$ y Ángela M. Ríos-Gallardo ${ }^{1}$ \\ ${ }^{1}$ Institución Universitaria Politécnico Grancolombiano, Bogotá, Colombia \\ ${ }^{2}$ Universidad Surcolombiana, Neiva, Colombia \\ ${ }^{3}$ Universidad de San Buenaventura, Medellín, Colombia
}

\begin{abstract}
Interventions for improving psychological health and cognitive, emotional and behavioral processes in children and adolescents who are victims of armed conflict. A systematic review
\end{abstract}

\begin{abstract}
Armed conflicts are related to social, economic, political and cultural difficulties that directly affect people who find themselves in the most vulnerable situations. Millions of children live in countries immersed in armed conflict and have been victims of forced migration and family disintegration and witnesses to traumatic events. The goal of this review was to assess the quality and efficacy of psychological treatments aimed at children and adolescents who are victims of armed conflict. The existing literature from January 2009 to December 2019 was analyzed conducting a systematic search of the Cochrane, Scopus, WoS, ProQuest, Pubmed, ScienceDirect and PsycINFO databases. A growing interest of scientific research in psychological interventions in humanitarian crises contexts is highlighted. There are quality clinical trials that include representative samples, follow-up assessment and treatments that involve the child's family as the key to possible positive changes at cognitive, emotional and behavioral level.
\end{abstract}

Keywords: Armed conflict; psychological intervention; children and adolescents; systematic review.

Resumen: Los conflictos armados están relacionados con dificultades sociales, económicas, políticas y culturales que afectan directamente a las personas en situaciones más vulnerables. Millones de niños viven en países inmersos en el conflicto armado y han sido víctimas de migración forzosa, desintegración familiar y testigos de eventos traumáticos. El objetivo de esta revisión fue evaluar la calidad y eficacia de los tratamientos psicológicos dirigidos a niños y adolescentes víctimas de conflicto armado. Se analizó la literatura existente desde enero de 2009 hasta diciembre de 2019 mediante una búsqueda sistemática en las bases de datos Cochrane, Scopus, WoS, ProQuest, Pubmed, Sciencedirect y PsycINFO. Se destaca un interés creciente de la investigación científica por la intervención psicológica en contextos de crisis humanitarias. Existen ensayos clínicos de calidad con la inclusión de muestras representativas, evaluaciones de seguimiento y tratamientos que incluyen la familia del niño como eje de posibles cambios positivos a nivel cognitivo, emocional y comportamental.

Palabras clave: Conflicto armado; intervención psicológica; niños y adolescentes; revisión sistemática.

\section{Introducción}

Los conflictos armados están relacionados con dificultades sociales, económicas, políticas y culturales que afectan

Recibido: 14 de febrero 2020; aceptado: 14 de julio 2020

Correspondencia: Cristian Villanueva-Bonilla, Institución Universitaria Politécnico Grancolombiano, Calle 57 No. 03-00 este, Bogotá, Colombia, Correo-e: cristian.villanueva@usco.edu.co directamente a las personas en situaciones más vulnerables. Millones de niños viven en países inmersos en el conflicto armado y han sido víctimas de migración forzosa, desintegración familiar y testigos de eventos traumáticos (Jordans et al., 2016). Los niños y adolescentes expuestos a situaciones de violencia corren un alto riesgo de desarrollar problemas de salud mental y necesitan recibir atención temprana no solo hacia sus dificultades físicas, económicas o sociales 
sino también ante posibles trastornos psicológicos (Betancourt et al., 2013a). En los últimos años ha habido un número considerable de estudios que investigan el estado de la salud mental de niños y adolescentes en contextos de guerra y otros que se han centrado en intervenir en esta población desde enfoques educativos, familiares o comunitarios para disminuir las dificultades psicológicas asociadas a estas problemáticas (Betancourt et al., 2013a; Betancourt, et al., 2013b; Jordans, et al., 2009; Tol et al., 2013).

El conflicto armado y las estadísticas de migración forzosa evidencian la difícil situación de salud pública a nivel mundial. En el año 2018 aproximadamente 4.9 millones de personas se vieron obligadas a dejar su hogar como consecuencia de la guerra (Norwegian Refugee Council, 2019) y países como Afganistán, Siria, Sudan, Congo, Myanmar, Iraq, Colombia y Vietnam presentan la cifra más alta de refugiados por conflicto armado (Norwegian Refugee Council, 2019). Uno de los grupos más afectados en el marco del conflicto son los menores de edad. Los niños y adolescentes se enfrentan a la separación de sus padres por desaparición forzosa o muerte, al abandono y a la carencia en la satisfacción de sus necesidades básicas, lo que les genera estados emocionales como ansiedad, miedo, rabia, desesperanza y depresión que pueden desembocar en conductas de agresividad, rechazo hacia los demás, inseguridad e impulsividad, entre otras (Castro-Camacho et al., 2019; Lee-Koo, 2018).

La investigación científica ha mostrado la estrecha relación que existe entre experimentar eventos traumáticos causados por la guerra y la alteración de la salud mental a diferentes niveles. Los eventos de guerra afectan al desarrollo cognitivo, emocional y comportamental de la población infanto-juvenil debido a la desestabilización familiar provocada por la exclusión de sus miembros, sus proyectos, sus patrones normativos y su educación. De esta manera, la familia, en un estado de vulnerabilidad, pierde su integridad por completo, eligiendo comportamientos de supervivencia que afectan la convivencia y el desarrollo cognitivo (Brown et al., 2017).

En este contexto, las intervenciones psicológicas están destinadas a prevenir y tratar los síntomas relacionados con el trauma en niños y adolescentes que han estado expuestos a conflictos armados (Dimitry, 2012). Estas intervenciones incluyen elementos psicoeducativos, psicodinámicos, de exposición, cognitivo-conductuales y de afrontamiento, los cuales pueden ayudar a niños o adolescentes con dificultades psicológicas a normalizar reacciones, procesar emocionalmente el trauma, comprender mejor su significado, desensibilizar los recuerdos relacionados con el trauma y manejar su ansiedad (Gillies et al., 2016). La inclusión de los padres puede promover la efectividad de estas terapias al mejorar su reconocimiento de los sín- tomas del trauma y optimizar su capacidad para gestionar la angustia emocional de sus hijos y los problemas de conducta relacionados (Purgato et al., 2018).

Los niños y adolescentes que han experimentado eventos traumáticos tienen un alto riesgo de desarrollar trastorno de estrés postraumático (TEPT), depresión, ansiedad y otros problemas psicológicos y sociales. Los conflictos armados impactan a una gran parte de la población mundial generando pobreza, violencia de género y marginación social (Norwegian Refugee Council, 2019). Es importante tener en cuenta que la aplicación de tratamientos con eficacia tiene el potencial de mejorar el funcionamiento psicológico y el bienestar individual.

Aunque se han realizado revisiones sistemáticas para examinar la efectividad de las intervenciones psicológicas dirigidas a niños y adolescentes expuestos a trauma (Gillies et al., 2016), los autores de este artículo no tienen conocimiento de la existencia de ninguna revisión sistemática que hasta la fecha haya estudiado la efectividad de las intervenciones psicológicas para mejorar la salud mental de niños y adolescentes víctimas de conflicto armado. La revisión sistemática de Gillies et al. (2016) encontró evidencias parciales de la efectividad de las terapias psicológicas para disminuir síntomas del TEPT hasta un mes después del tratamiento, con una calidad de los estudios de baja a moderada y sin efectos concretos a largo plazo. Los autores encontraron que los estudios incluidos en la revisión implicaban estresores diferentes como el abuso sexual, el maltrato físico, la violencia interpersonal, la enfermedad terminal y la violencia comunitaria. Por esta razón, la comparación entre las intervenciones y las diferencias significativas encontradas en dichos estudios pudo generar conclusiones que no describían en forma precisa la realidad de los participantes.

En resumen, dado el amplio impacto de los conflictos armados en la salud mental, esta revisión tuvo como objetivo proporcionar una evaluación de la calidad y eficacia de los estudios sobre tratamientos enfocados en mejorar la salud psicológica y los procesos cognitivos, emocionales y comportamentales en niños y adolescentes víctimas de conflicto armado.

\section{Método}

Esta revisión se desarrolló siguiendo las pautas y orientaciones de la guía PRISMA (Moher et al., 2009).

\section{Criterios de elegibilidad}

Participantes. Estudios que incluyan niños y adolescentes víctimas de conflicto armado (entre el nacimiento y los 18 años). Los estudios también pueden incluir a las 
familias y/o cuidadores de los participantes como un apoyo o complemento a la intervención.

Intervención. Se incluye cualquier ensayo clínico aleatorizado (ECA) centrado en abordar los procesos cognitivos, emocionales y/o comportamentales en niños y adolescentes víctimas de conflicto. Se incluyen todo tipo de terapias psicológicas como, por ejemplo, terapia cognitivo-conductual, capacitación o formación a padres y familiares, terapia de exposición narrativa para niños, intervención escolar, terapia cognitiva conductual centrada en el trauma, programa de deporte para el desarrollo y grupos de asesoramiento.

Comparación. Como grupo de comparación se incluyen grupos control (tratamiento habitual, controles en lista de espera o ningún tratamiento) así como intervenciones de terapia farmacológica o ECAs que incluyan dos o más grupos de tratamiento con terapias psicológicas.

Resultados. Los estudios se incluyen si presenta en sus resultados mediciones de psicopatología (síntomas de TEPT, trastornos de ansiedad, depresión, entre otras), procesos cognitivos (atención, memoria, función ejecutiva, lenguaje, entre otras), problemas conductuales y emocionales (empatía comportamental, resolución de problemas, agresión, conducta prosocial, inseguridad, entre otras), redes familiares, escolares y sociales (impacto en el rendimiento escolar, deterioro funcional, vida familiar, entre otras), resiliencia, estrategias de afrontamiento y esperanza.

Diseño de estudio. Se incluyen ECAs publicados entre enero de 2009 y diciembre de 2019, escritos en inglés o español y publicados en revistas con proceso de revisión por pares.

\section{Fuentes de información y búsqueda}

Se identificaron los estudios mediante una búsqueda sistemática en las siguientes bases de datos electrónicas: Cochrane, Scopus, Web of Science, ProQuest, Pubmed, Sciencedirect, PsycINFO. La búsqueda principal se realizó en agosto de 2019 y se actualizó por última vez en junio de 2020. La sintaxis de búsqueda fue la siguiente:

TITLE-ABS-KEY((child* OR adolescent OR teen*) AND (intervention OR treatment OR training OR coaching OR therapy) AND (cogniti* OR emotion* OR conduct OR behavio* OR empathy OR «theory of mind» OR mentalizing OR «social skills» OR «social abilities» OR psychological) AND («armed conflict» OR «armed vio- lence» OR «conflict affected» OR post-conflict OR «war experiences» OR «war affected» OR war) AND («clinical trial» OR «controlled trial»)) AND (LIMIT-TO (PUBYEAR, 2019) OR LIMIT-TO ( PUBYEAR, 2018) OR LIMIT-TO (PUBYEAR, 2017) OR LIMIT-TO (PUBYEAR, 2016) OR LIMIT-TO (PUBYEAR, 2010) OR LIMIT-TO (PUBYEAR, 2015) OR LIMIT-TO (PUBYEAR,2014) OR LIMIT-TO (PUBYEAR, 2013) OR LIMIT-TO (PUBYEAR, 2012) OR LIMIT-TO (PUBYEAR, 2011) OR LIMIT-TO ( PUBYEAR, 2009) )

Términos como «empathy», «theory of mind» «mentalizing» $\mathrm{y}$ «social skills» fueron incluidos como palabras clave debido al conocimiento de los autores sobre la importancia de dichas variables en los procesos de tratamiento emocional, cognitivo y comportamental de niños y adolescentes expuestos a situaciones de violencia. No se excluyeron estudios que de forma complementaria incluían a familiares y cuidadores ya que se consideró su relevancia como apoyo de la intervención a los menores.

Se importaron todas las referencias obtenidas de las bases de datos a Endnote y se eliminaron los duplicados. Dos investigadores examinaron de forma independiente todos los títulos y resúmenes utilizando los criterios de elegibilidad especificados y recuperaron y evaluaron los documentos de texto completo en el caso de los estudios potencialmente elegibles. Se resolvió cualquier discrepancia en cada etapa mediante discusión o consultando a un tercer investigador en caso de no llegar a un acuerdo.

\section{Variables de codificación}

Se extrajeron las siguientes variables de cada uno de los estudios: información general sobre el estudio (título, autores, año de publicación, país de publicación), método (diseño del estudio, asignación a grupos de tratamiento, duración del estudio), participantes (entorno, método de reclutamiento, pérdida de muestra, detalles de diagnóstico relevantes, edad, sexo, asignación de subgrupos), intervención (consideraciones y componentes relacionados con la intervención incluyendo las bases teóricas, la duración, la frecuencia de las sesiones, la administración individual o grupal, la formación del personal que administra la intervención, las medidas y escalas de resultados) y resultados (primarios y secundarios).

\section{Evaluación del riesgo de sesgo en los estudios incluidos}

Dos investigadores evaluaron de forma independiente cada estudio para determinar el riesgo de sesgo. Los desacuerdos se resolvieron mediante discusión y cuando fue necesario un tercer investigador evaluó de forma independiente el estudio para llegar a un acuerdo. La eva- 
luación de «riesgo de sesgo» para los ECAs utilizados en esta revisión se realizó utilizando los criterios recomendados en el Manual Cochrane para revisiones sistemáticas (Higgins y Green, 2011): 1) Generación de secuencia aleatoria; 2) Ocultamiento de la asignación; 3) Cegamiento de participantes y personal; 4) Cegamiento de los evaluadores; 5) Datos de resultado incompletos; 6) Notificación selectiva de resultados; y 7) Otro sesgo.

\section{Resultados}

Se identificaron 551 artículos, de los que finalmente se seleccionaron 27 artículos que cumplían con los criterios de inclusión. La Figura 1 resume el proceso de selección de los estudios.

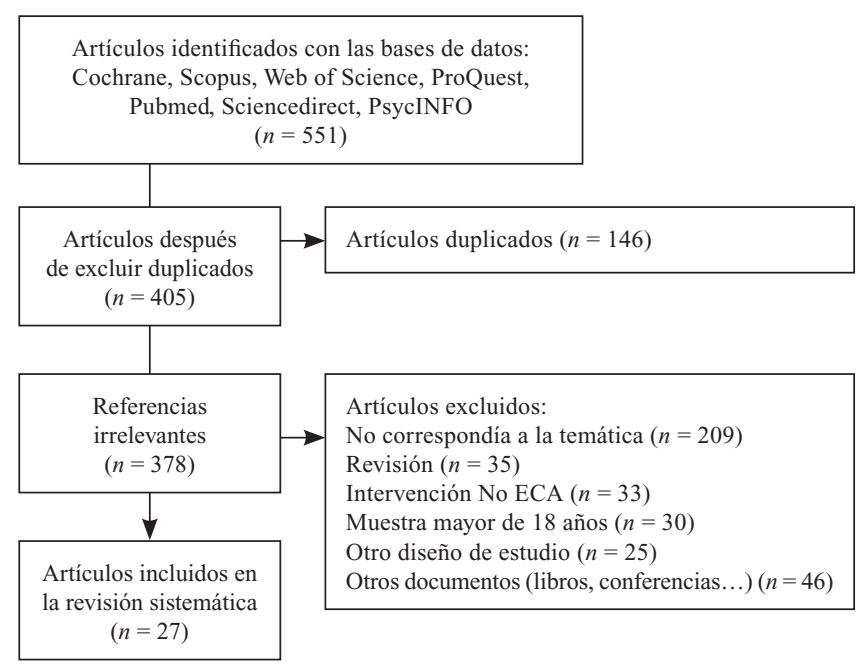

Figura 1. Diagrama de flujo del proceso de selección de los estudios.

Los estudios corresponden a ECAs de intervenciones enfocadas en mejorar la salud psicológica y los procesos cognitivos, emocionales y comportamentales de niños y adolescentes víctimas de conflicto armado. Se incluyeron estudios que evaluaban intervenciones de tipo individual (7 intervenciones), familiar (4 intervenciones) y social ( 8 intervenciones). En la Tabla 1 se presenta el resumen o tabla de codificación que incluye las características y resultados de los estudios incluidos en la presente revisión.

\section{Intervenciones individuales}

Como se evidencia en la Tabla 2, se identificaron 7 intervenciones de tipo individual en 12 estudios ${ }^{1}$. Las in-

\footnotetext{
${ }^{1}$ Barron et al., 2016; Barron et al., 2013; Catani et al., 2009; Dawson et al., 2018; Kalantari et al., 2012; Lange-Nielsen et al., 2012; McMu-
}

tervenciones identificadas son: enseñanza de técnicas de recuperación (ETR) (Barron et al., 2016; Barron et al., 2013; Ooi et al., 2016; Qouta et al., 2012), terapia de exposición narrativa para niños (KIDNET) (Catani et al., 2009), terapia de exposición narrativa para ex combatientes (FORNET) (Robjant et al., 2019), terapia de meditación-relajación (MED-RELAX) (Catani et al., 2009), terapia cognitivo conductual centrada en el trauma (TCC-CT) (Dawson et al., 2018; McMullen et al., 2013; Murray et al., 2015; O'Callaghan et al., 2013), terapia de resolución de problemas (RP) (Dawson et al., 2018) y «Writing for Recovery» (WfR) (Kalantari et al., 2012; Lange-Nielsen et al., 2012).

Las intervenciones más citadas corresponden a intervenciones de tipo individual basadas en el modelo cognitivo-conductual (TCC-CT y ETR). Dos de los 4 estudios de TCC-CT comparan la intervención con un grupo control (McMullen et al., 2013; O'Callaghan et al., 2013) y los 2 restantes utilizan la RP y servicios comunitarios como medio de comparación (Dawson et al., 2018; Murray et al., 2015). McMullen et al. (2013) y O'Callaghan et al. (2013) evalúan TEPT, funcionamiento psicosocial, depresión, ansiedad, problemas de conducta y comportamiento prosocial reportando diferencias significativas a favor del tratamiento en cada una de las variables estudiadas. Por su parte, Dawson et al. (2018) y Murray et al. (2015) también reportaron reducciones significativas en variables como el TEPT, el deterioro funcional y la expresión de la ira debido a TCC-CT y RP.

Los estudios que abordan ETR como estrategia de intervención para mejorar las dificultades asociadas a conflictos armados demuestran cambios significativos favorables en TEPT, depresión, disociación peritraumática y salud mental en general (Barron et al., 2016; Barron et al., 2013; Ooi et al., 2016; Qouta et al., 2012); además identifican que la mejoría en los síntomas de depresión se mantuvieron a los tres meses de seguimiento (Ooi et al., 2016).

Kalantari et al. (2012) y Lange-Nielsen et al. (2012) utilizaron la terapia WfR para disminuir síntomas asociados a TEPT, depresión, ansiedad y duelo traumático en niños y adolescentes en contextos vulnerables. Los resultados evidencian disminuciones significativas en TEPT y duelo traumático al final de la intervención y en depresión en la evaluación de seguimiento. Por último, Catani et al. (2009) y Robjant et al. (2019) evaluaron la eficacia de tres tratamientos (KIDNET, MED-RELAX y FORNET) sobre el TEPT, agresión, depresión, relaciones sociales, vida familiar y la satisfacción general con

llen et al., 2013; Murray et al., 2015; O'Callaghan et al., 2013; Ooi et al., 2016; Qouta et al., 2012; Robjant et al., 2019. 


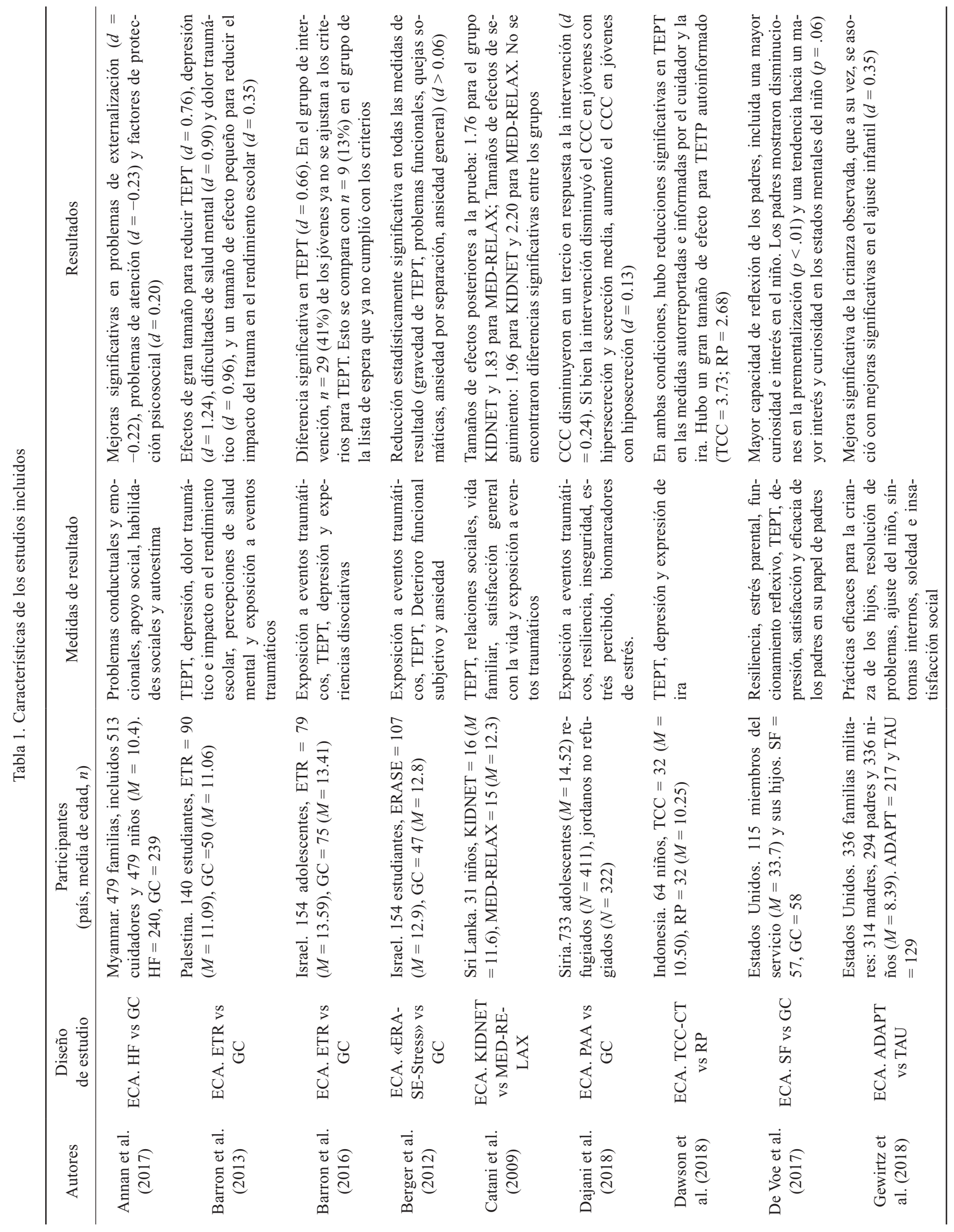




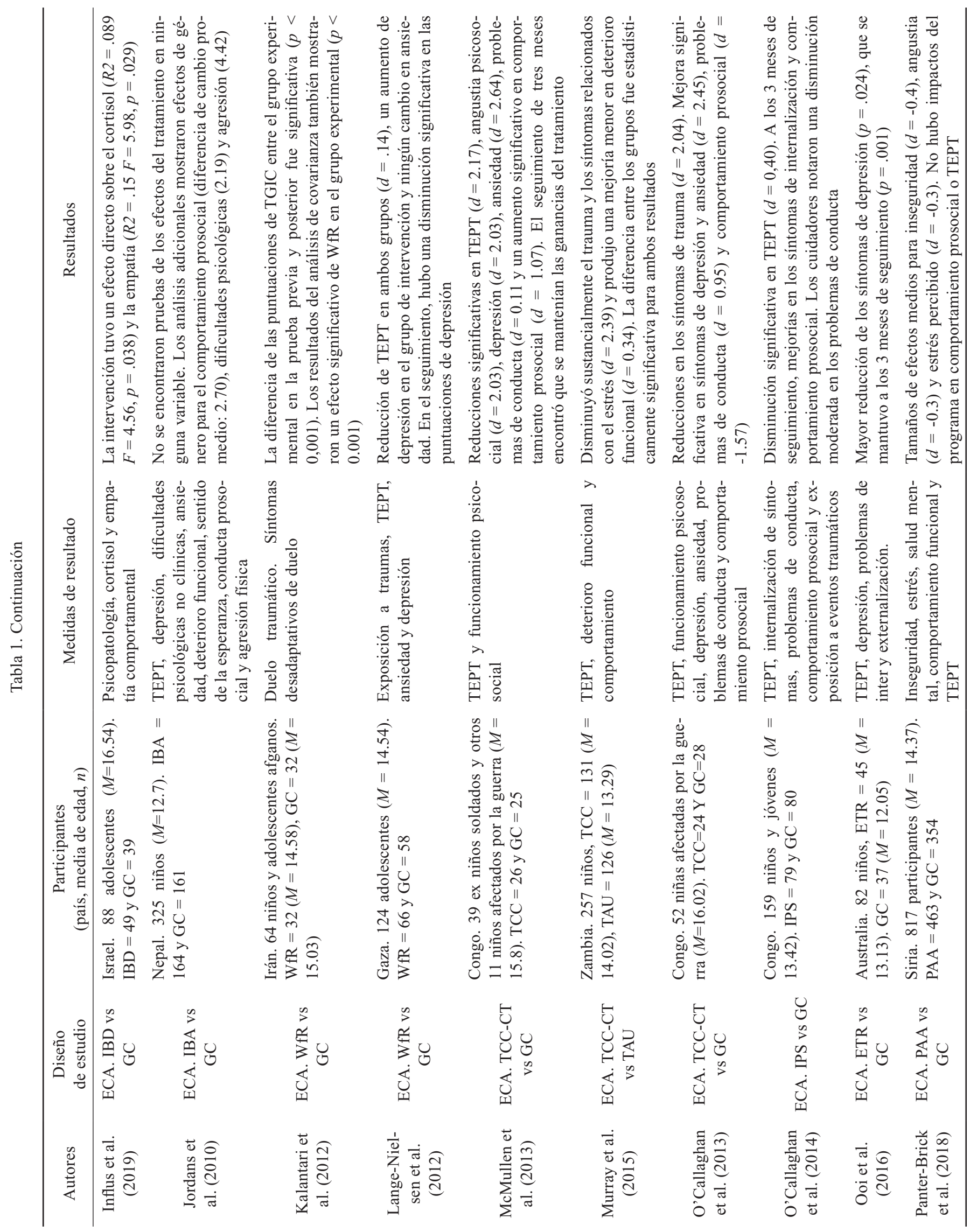




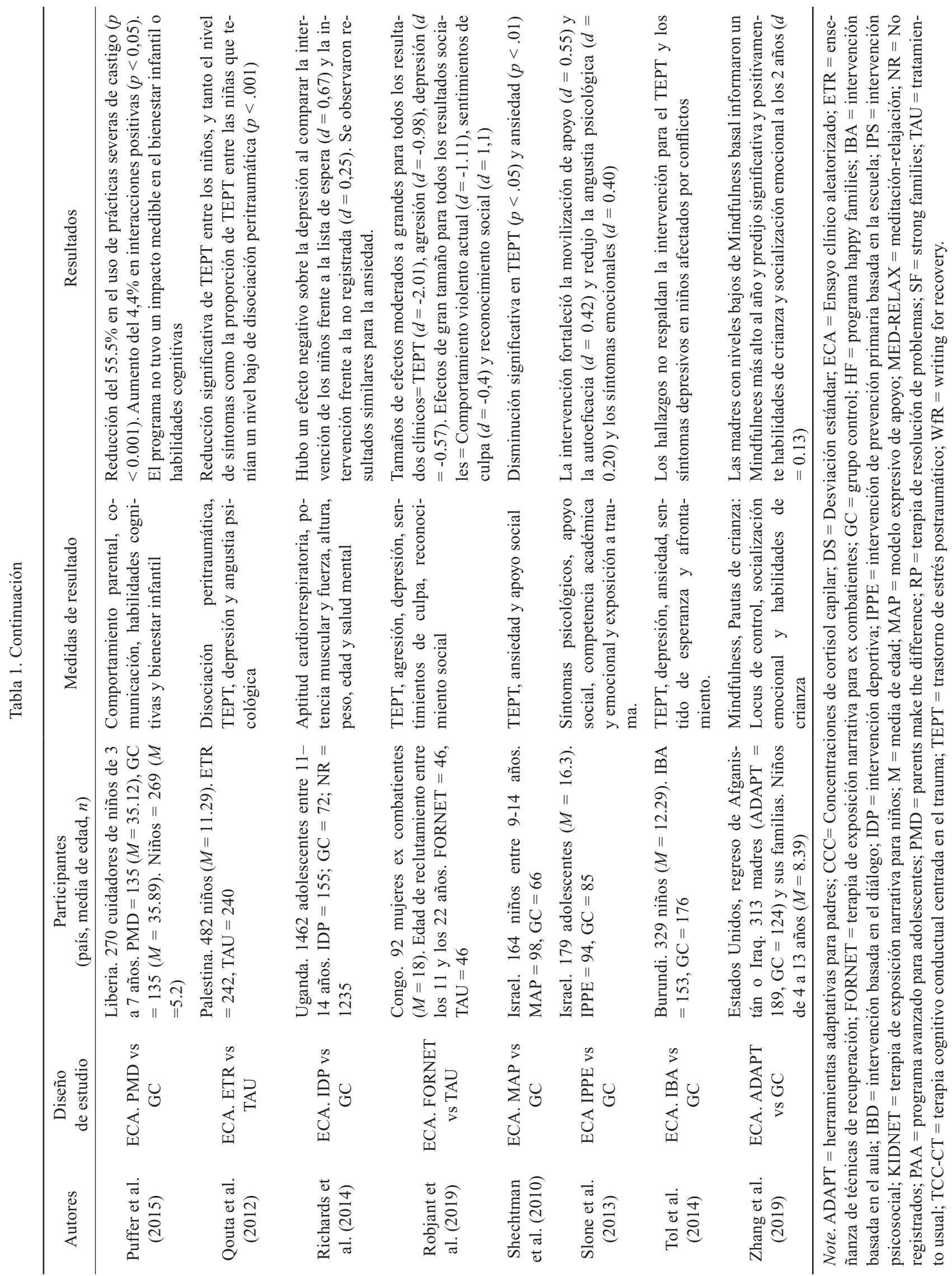


la vida. Los autores encontraron que los tratamientos fueron efectivos para mejorar síntomas asociados al TEPT, agresión, depresión y variables sociales que se mantuvieron a los 6 meses de seguimiento.

\section{Intervenciones familiares}

Como se evidencia en la Tabla 2, se identificaron 4 intervenciones de tipo familiar en 5 estudios $^{2}$. Las intervenciones identificadas son: Programa «Happy Families» (HF) (Annan et al., 2017), «Strong Families» (SF) (De Voe et al., 2017), herramientas adaptativas para padres (ADAPT) (Gewirtz et al., 2018; Zhang et al., 2019) y «Parents Make the Difference» (PMD) (Puffer et al., 2015).

Los programas SF, HF, ADAPT y PMD utilizan estrategias enfocadas en mejorar habilidades relacionadas con la crianza positiva, comunicación, empatía y habilidades sociales en general que permiten la disminución de dificultades asociadas al conflicto en la el núcleo familiar. Annan et al. (2017) y De Voe et al. (2017) encontraron mejorías significativas en problemas de externalización, atención, factores de protección psicosocial, funcionamiento reflexivo y mentalización después de la implementación de los programas de intervención. En este sentido, Gewirtz et al. (2018), Puffer et al. (2015) y Zhang et al. (2019) encontraron diferencias a favor de las intervenciones en habilidades de crianza e interacciones positivas entre el cuidador y el niño; sin embargo, no se presentaron resultados positivos en variables como el bienestar infantil y las habilidades cognitivas (Puffer et al., 2015).

\section{Intervenciones sociales}

Como se evidencia en la Tabla 2, se identificaron 8 intervenciones de tipo social en 10 estudios $^{3}$. Las intervenciones sociales o escolares identificadas son: «ERASE-Stress» (Berger et al., 2012), programa avanzado para adolescentes (PAA) (Dajani et al., 2018; Panter-Brick et al., 2018), intervención Basada en el Aula (IBA) (Jordans et al., 2010; Tol et al., 2014), intervención Psicosocial (IPS) (O'Callaghan et al., 2014), intervención deportiva (IDP) (Richards et al., 2014), modelo expresivo de apoyo (MAP) (Shechtman y Mor, 2010),

\footnotetext{
${ }^{2}$ Annan et al., 2017; De Voe et al., 2017; Gewirtz et al., 2018; Puffer et al., 2015; Zhang et al., 2019.

3 Berger et al., 2012; Dajani et al., 2018; Influs et al., 2019; Jordans et al., 2010; O'Callaghan et al., 2014; Panter-Brick et al., 2018; Richards et al., 2014; Shechtman y Mor, 2010; Slone et al., 2013; Tol et al., 2014.
}

intervención de prevención primaria basada en la escuela (IPPE) (Slone et al., 2013) e intervención basada en el diálogo (IBD) (Influs et al., 2019).

La intervención PAA es una de las intervenciones de tipo social más citada en la presente revisión (Dajani et al., 2018; Panter-Brick et al., 2018). Dicho programa se basa en elementos de seguridad, apoyo social y actividades grupales para adolescentes con dificultades derivadas de la guerra. Los resultados muestran efectos positivos en inseguridad, angustia, estrés percibido, nivel de cortisol y salud mental general; sin embargo, no se evidencian mejorías en comportamiento prosocial y TEPT (Dajani et al., 2018; Influs et al., 2019; Panter-Brick et al., 2018). Por su parte, O'Callaghan et al. (2014) reportaron cambios significativos en los síntomas de TEPT, problemas de internacionalización y comportamiento prosocial a los 3 meses de seguimiento. Resultados similares fueron encontrados para TEPT y ansiedad en una intervención enfocada en la expresión y exploración de sentimientos en un clima emocional de apoyo (Shechtman y Mor, 2010) y para depresión y ansiedad en una intervención basada en la comunidad para promover salud mental y consolidación de paz (Richards et al., 2014).

Otras intervenciones tomaron como eje el contexto escolar (ERASE-Stress, IBA y IPPE). En este sentido, Jordans et al. (2010) y Tol et al. (2014) no encontraron evidencias del tratamiento sobre ninguna de las variables medidas (TEPT, depresión, ansiedad y deterioro funcional, entre otras), mencionando que los posibles beneficios están asociados a variables como la edad, el género y el funcionamiento familiar. En cambio, Berger et al. (2012) y Slone et al. (2013) reportaron efectos positivos y significativos a favor de las intervenciones en TEPT, problemas funcionales, quejas somáticas, ansiedad, apoyo social y autoeficacia.

\section{Riesgo de sesgo en los estudios incluidos}

Para ver representaciones gráficas del riesgo general de sesgo en los estudios incluidos, consulte la Figura 2 y la Figura 3.

Asignación. Los investigadores describieron la generación de una secuencia aleatoria que se considera que conduce a un bajo riesgo de sesgo en 17 estudios $^{4}$, riesgo

\footnotetext{
${ }^{4}$ Annan et al., 2017; Barron et al., 2016; Berger et al., 2012; Catani et al., 2009; Dawson et al., 2018; Influs et al., 2019; Jordans et al., 2010; Lange-Nielsen et al., 2012; McMullen et al., 2013; Murray et al., 2015; O'Callaghan et al., 2014; O'Callaghan et al., 2013; Ooi et al., 2016; Puffer et al., 2015; Qouta et al., 2012; Richards et al., 2014; Robjant et al., 2019.
} 


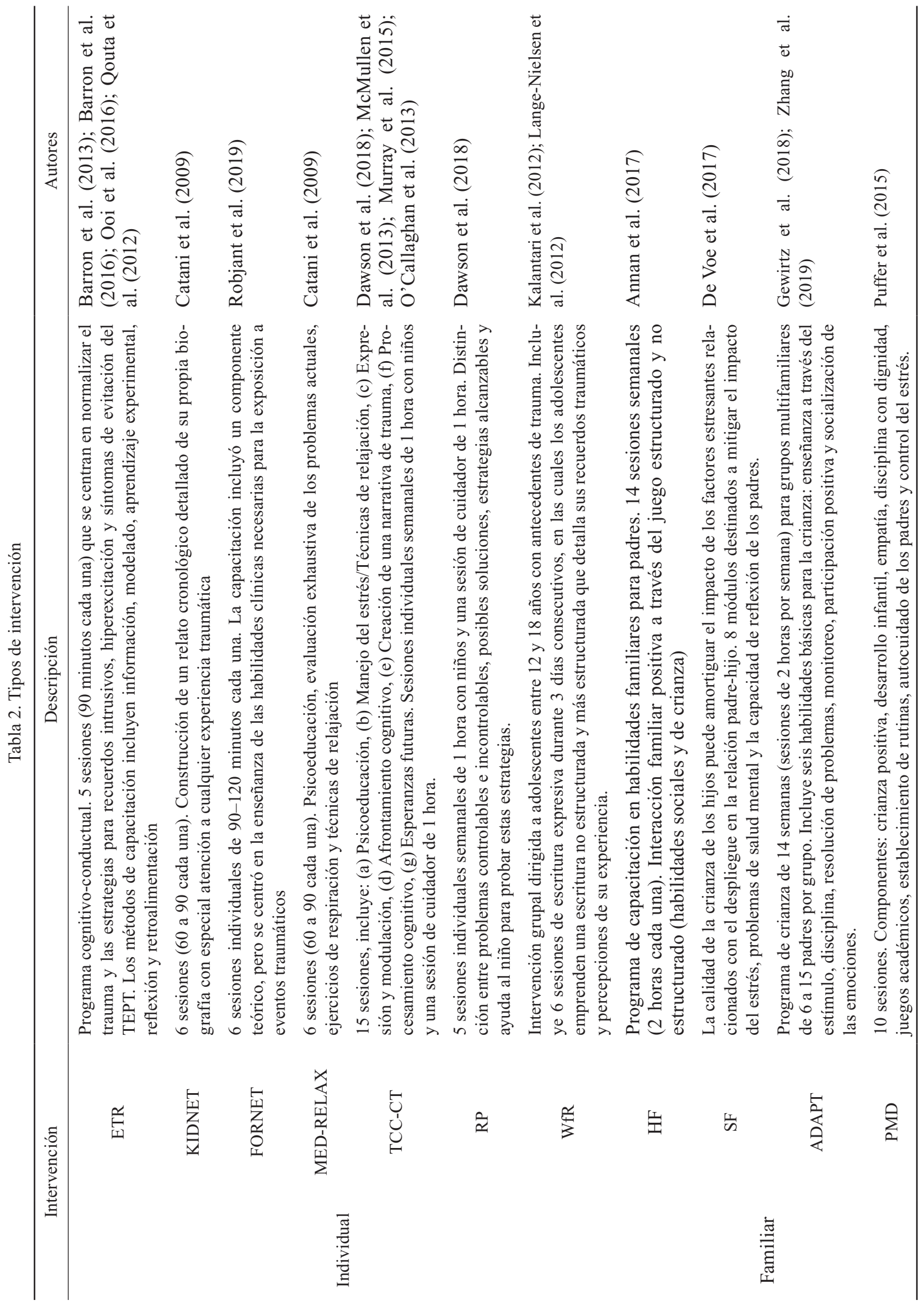




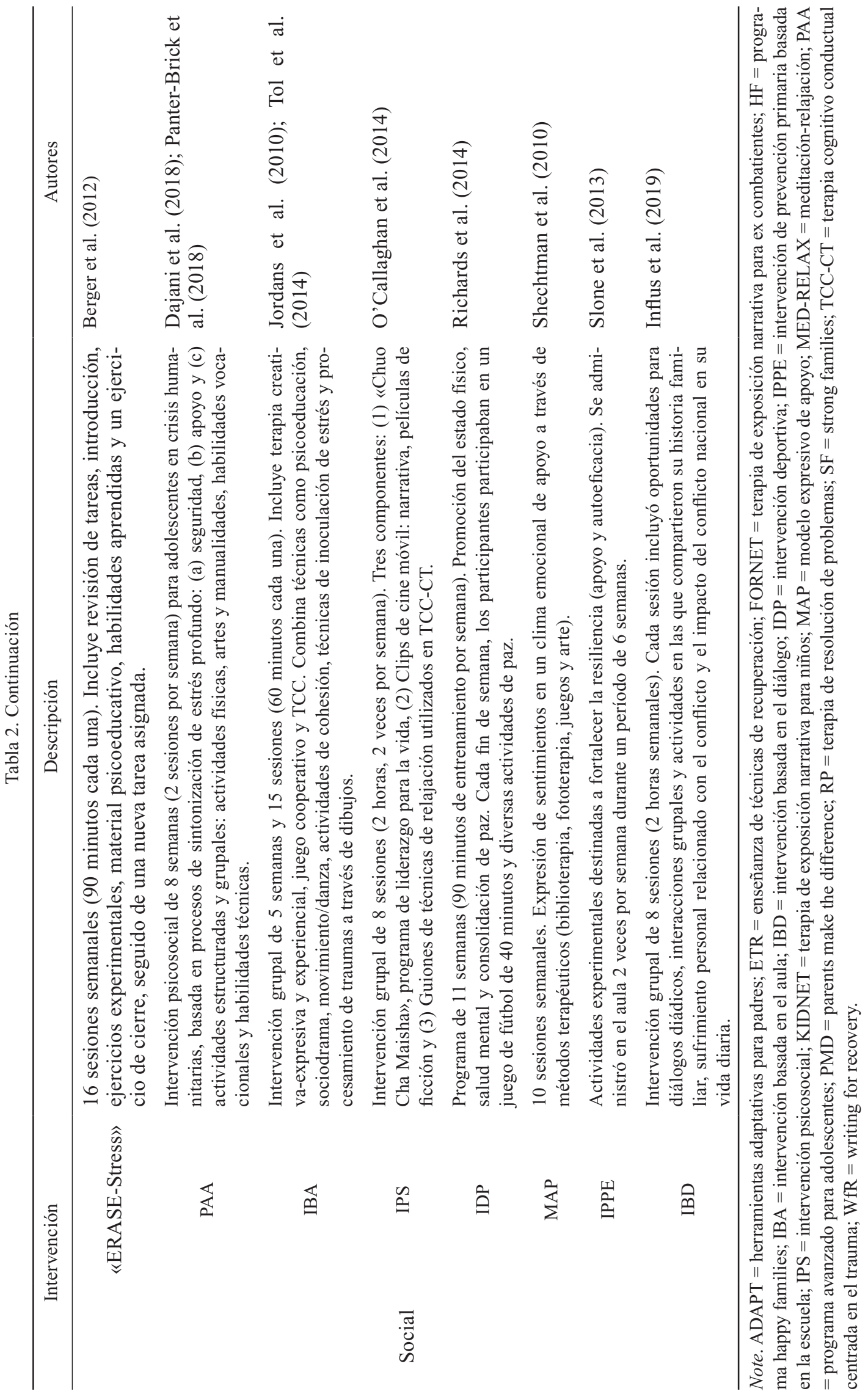




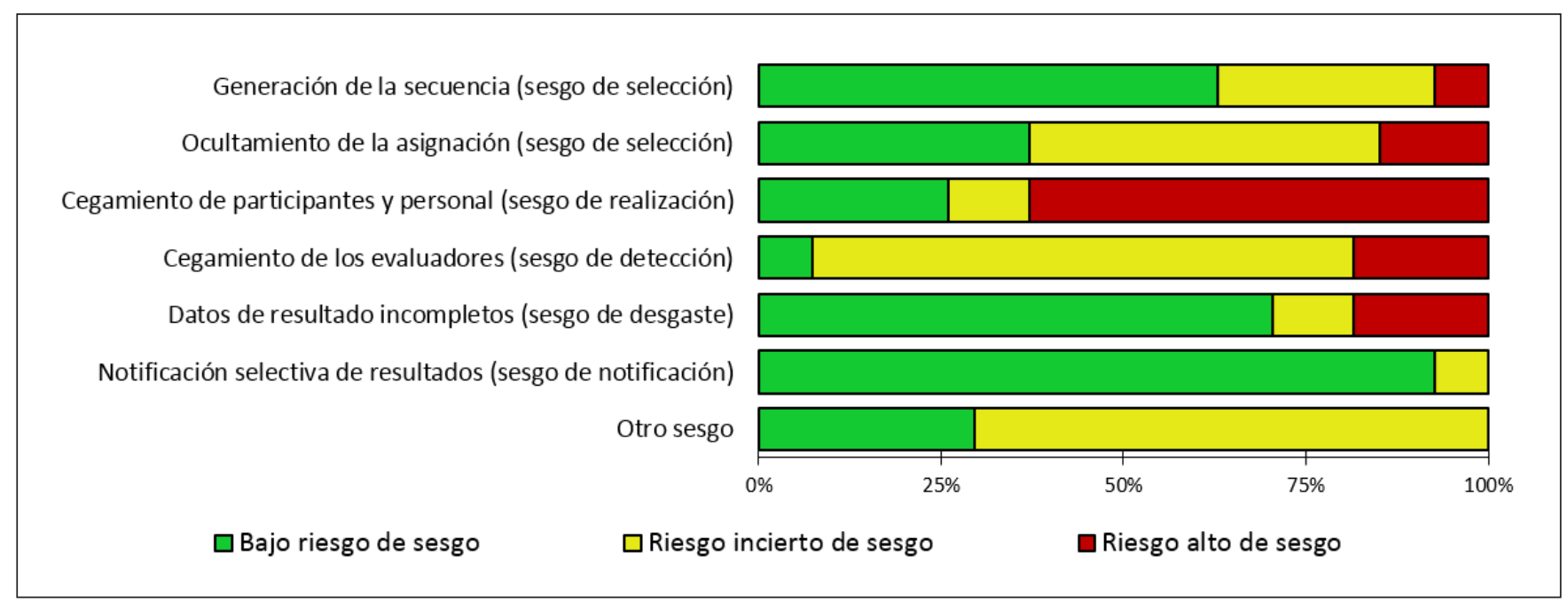

Figura 2. Riesgo de sesgo de los estudios incluidos, porcentaje por criterio según Cochrane (Higgins y Green, 2011)

incierto de sesgo en 8 estudios ${ }^{5}$ y alto riesgo de sesgo en 2 estudios (Barron et al., 2013; Panter-Brick et al., 2018). Con respecto al ocultamiento de la asignación, se consideró que 10 de los ensayos incluidos tenían bajo riesgo ${ }^{6}$, 13 estudios con riesgo incierto ${ }^{7}$ y 4 estudios con riesgo alto de sesgo (Barron et al., 2016; Barron et al., 2013; Lange-Nielsen et al., 2012; Panter-Brick et al., 2018).

Cegamiento. Los participantes probablemente sabían si habían sido asignados a un grupo de intervención o un grupo control en 17 ensayos, que fueron calificados como de alto riesgo de sesgo ${ }^{8}$ mientras que 7 estudios fueron clasificados con bajo riesgo ${ }^{9}$ y los demás ensayos se calificaron con riesgo incierto. Los resultados primarios fueron autoinformados en la mayoría de los estudios (20) y fueron calificados como de riesgo incierto de sesgo ${ }^{10}, 2$ estudios fueron clasificados como de bajo riesgo por el método de ocultamiento de resultados utilizado (Dawson et al., 2018; O'Callaghan et al.,

\footnotetext{
${ }_{5}^{5}$ Dajani et al., 2018; De Voe et al., 2017; Gewirtz et al., 2018; Kalantari et al., 2012; Shechtman \& Mor, 2010; Slone et al., 2013; Tol et al., 2014; Zhang et al., 2019.

${ }^{6}$ Dawson et al., 2018; McMullen et al., 2013; Murray et al., 2015; O'Callaghan et al., 2014; O'Callaghan et al., 2013; Ooi et al., 2016; Qouta et al., 2012; Richards et al., 2014; Robjant et al., 2019; Slone et al., 2013.

${ }^{7}$ Annan et al., 2017; Berger et al., 2012; Catani et al., 2009; Dajani et al., 2018; De Voe et al., 2017; Gewirtz et al., 2018; Influs et al., 2019; Jordans et al., 2010; Kalantari et al., 2012; Puffer et al., 2015; Shechtman \& Mor, 2010; Tol et al., 2014; Zhang et al., 2019.

${ }^{8}$ Barron et al., 2016; Barron et al., 2013; Berger et al., 2012; Dajani et al., 2018; De Voe et al., 2017; Gewirtz et al., 2018; Influs et al., 2019; Jordans et al., 2010; Lange-Nielsen et al., 2012; O'Callaghan et al., 2014; O'Callaghan et al., 2013; Ooi et al., 2016; Panter-Brick et al., 2018; Richards et al., 2014; Shechtman \& Mor, 2010; Tol et al., 2014; Zhang et al., 2019.
}

2014) y los demás ensayos se calificaron con alto riesgo de sesgo.

Datos de resultado incompletos. Un total de 19 estudios fueron calificados con riesgo de sesgo de deserción bajo porque reportaron consistentemente las pérdidas de muestra durante el tratamiento ${ }^{11}, 5$ ensayos fueron calificados de alto riesgo debido a que la pérdida muestral supera el 20\% (Gewirtz et al., 2018; Influs et al., 2019; McMullen et al., 2013; Tol et al., 2014; Zhang et al., 2019). Los demás estudios se calificaron con riesgo incierto de sesgo.

Notificación selectiva de los resultados. 25 estudios presentan una descripción completa de las variables de resultado $^{12}$ y los 2 ensayos restantes se calificaron con riesgo incierto de sesgo debido a que presentan una descripción parcial de algunas variables (Murray et al., 2015; O’Callaghan et al., 2014).

\footnotetext{
9 Annan et al., 2017; Catani et al., 2009; Dawson et al., 2018; McMullen et al., 2013; Murray et al., 2015; Robjant et al., 2019; Slone et al., 2013. ${ }^{10}$ Annan et al., 2017; Barron et al., 2016; Barron et al., 2013; Berger et al., 2012; Gewirtz et al., 2018; Influs et al., 2019; Kalantari et al., 2012; Lange-Nielsen et al., 2012; McMullen et al., 2013; Murray et al., 2015; O'Callaghan et al., 2013; Ooi et al., 2016; Panter-Brick et al., 2018; Qouta et al., 2012; Richards et al., 2014; Robjant et al., 2019; Shechtman \& Mor, 2010; Slone et al., 2013; Tol et al., 2014; Zhang et al., 2019.

11 Annan et al., 2017; Barron et al., 2016; Barron et al., 2013; Berger et al., 2012; Catani et al., 2009; Dawson et al., 2018; De Voe et al., 2017; Jordans et al., 2010; Kalantari et al., 2012; Lange-Nielsen et al., 2012; Murray et al., 2015; O'Callaghan et al., 2014; O'Callaghan et al., 2013; Ooi et al., 2016; Puffer et al., 2015; Qouta et al., 2012; Richards et al., 2014; Robjant et al., 2019; Slone et al., 2013.

12 Annan et al., 2017; Barron et al., 2016; Barron et al., 2013; Berger et al., 2012; Catani et al., 2009; Dajani et al., 2018; Dawson et al., 2018; De Voe et al., 2017; Gewirtz et al., 2018; Influs et al., 2019;
} 


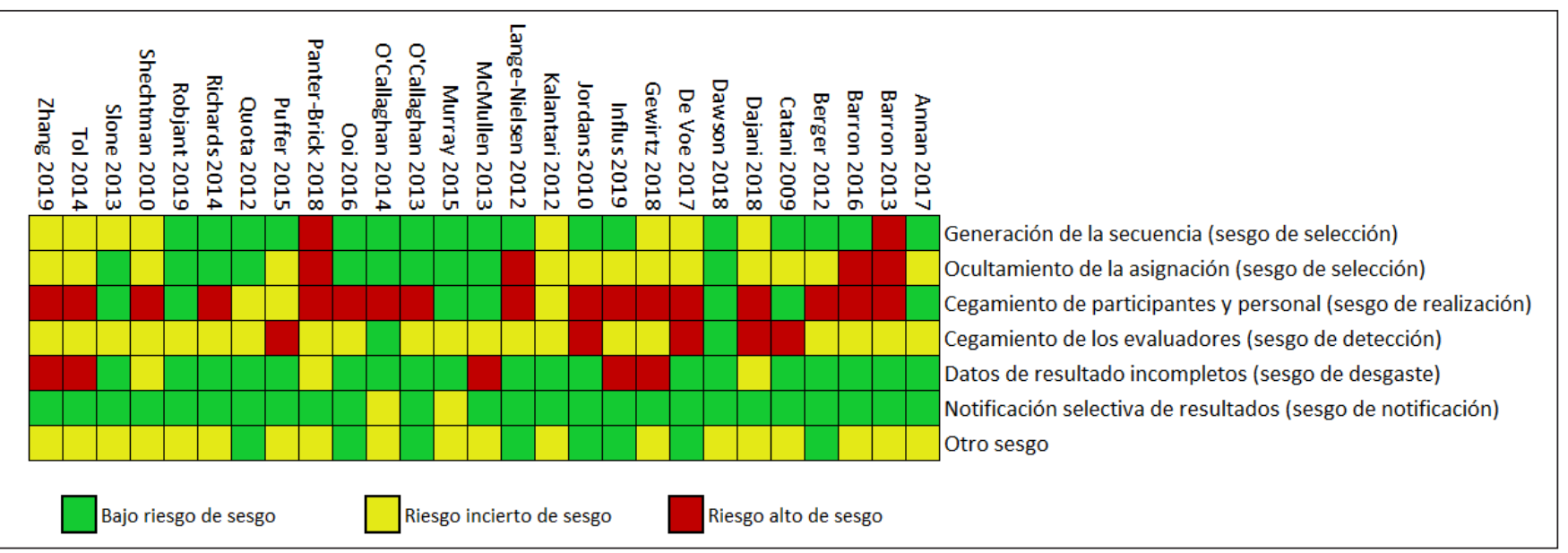

Figura 3. Riesgo de sesgo de los estudios incluidos según Cochrane (Higgins y Green, 2011)

Otro sesgo. Se tuvieron en cuenta la cualificación del terapeuta (profesionales de la psicología o áreas afines) y la fidelidad del tratamiento (método utilizado para corroborar la adecuada implementación del programa de intervención). 8 ensayos describieron y cumplieron apropiadamente las dos características en mención ${ }^{13}$ mientras que los demás estudios incumplieron con una de las dos características y fueron calificados con riego incierto.

\section{Discusión}

El objetivo de esta revisión fue evaluar la calidad y eficacia de los ECAs que evalúan intervenciones enfocadas en mejorar la salud psicológica y los procesos cognitivos, emocionales y comportamentales de niños y adolescentes víctimas de conflicto armado. Se incluyen estudios publicados entre enero de 2009 hasta diciembre de 2019. El número de artículos incluidos fue limitado $(n=27)$. No obstante, se han encontrado hallazgos importantes en relación a la efectividad de los tratamientos identificados sobre las variables de resultado (psicopatología, cognición y neurocognición, problemas conductuales y emocionales, redes familiares, escolares y sociales, resiliencia, afrontamiento y esperanza). Los estudios realizados en los últimos 10 años utilizan mayoritariamente el modelo cognitivo-conductual y son los que aportan una mayor efectividad en el tratamiento de niños y adolescentes víctimas de conflicto. Dichos tratamientos presentan como resultados principales la disminución de los síntomas asociados con TEPT, de-

Jordans et al., 2010; Kalantari et al., 2012; Lange-Nielsen et al., 2012; McMullen et al., 2013; O'Callaghan et al., 2013; Ooi et al., 2016; Panter-Brick et al., 2018; Puffer et al., 2015; Qouta et al., 2012; Richards et al., 2014; Robjant et al., 2019; Shechtman \& Mor, 2010; Slone et al., 2013; Tol et al., 2014; Zhang et al., 2019. presión, ansiedad y problemas de comportamiento, entre otros.

En cuanto a la calidad de las investigaciones, todos los estudios incluidos pueden considerarse relativamente robustos al ser ECAs. Otra fortaleza fue el bajo porcentaje de pérdida muestral que tuvo lugar en la mayoría de los estudios. En general, el sesgo de selección, desgaste y notificación en los ensayos incluidos proporcionaron detalles adecuados que permitieron un juicio positivo de su calidad en la mayoría de los casos. Por otro lado, se consideró que la mayoría de los ensayos tenían un alto riesgo de sesgo de realización ya que no incluían un tratamiento paralelo o placebo. Otro aspecto a destacar teniendo en cuenta el contexto de guerra y el difícil acceso a la población, fue el tamaño de las muestras, en la mayoría de los ensayos fueron adecuadas, 18 de los 24 estudios incluidos contaron con una muestra superior a 100 participantes y en diez de ellos fue superior a 200.

Los contextos donde se llevaron a cabo los tratamientos son complejos y los espacios y el tiempo limitados por lo que muchos estudios no incluían tratamientos de más de 15 sesiones (Annan et al., 2017; Barron et al., 2016; Catani et al., 2009; Dawson et al., 2018; Gewirtz et al., 2018; Influs et al., 2019; Jordans et al., 2010; Kalantari et al., 2012; O'Callaghan et al., 2014; Puffer et al., 2015; Robjant et al., 2019; Shechtman y Mor, 2010; Slone et al., 2013; Zhang et al., 2019). Aunque se tiene en cuenta el contexto mencionado, se hace necesaria la inclusión de un mayor número de sesiones que permitan mejores resultados sobre las variables de salud mental, aplicando la evi-

\footnotetext{
${ }^{13}$ Berger et al., 2012; De Voe et al., 2017; Influs et al., 2019; Jordans et al., 2010; Lange-Nielsen et al., 2012; O’Callaghan et al., 2013; Ooi et al., 2016; Qouta et al., 2012.
} 
dencia descrita en la literatura sobre el tiempo necesario y el número de sesiones ideal para generar resultados perdurables. Las investigaciones recomiendan tratamientos con 16 o más sesiones y con una duración de al menos 16 semanas. (Deblinger et al., 2011; Layne et al., 2008; Márquez et al., 2020; Oprel et al., 2018).

Asimismo, se identificó que pocos estudios realizaron evaluaciones de seguimiento mayores de 3 meses que permitieran determinar si los resultados encontrados se mantenían consistentes en el tiempo (Annan et al., 2017; Dajani et al., 2018; De Voe et al., 2017; Gewirtz et al., 2018; Qouta et al., 2012; Robjant et al., 2019; Zhang et al., 2019). También se resalta la escasez de estudios integrales que incluyan a los padres o cuidadores de los niños dentro del contexto de intervención, hecho que pudo haber generado cambios positivos en variables familiares y sociales (Annan et al., 2017; De Voe et al., 2017; Gewirtz et al., 2018; Puffer et al., 2015; Zhang et al., 2019).

Las intervenciones individuales (ETR, KIDNET, FORNET, MED-RELAX, TCC-CT, RP, WfR) tuvieron como objetivo primordial disminuir aquellas dificultades psicológicas asociadas al evento traumático específicamente en los niños y adolescentes víctimas, sin la inclusión de un familiar o un cuidador y en espacios donde la socialización entre pares es un resultado secundario. Dichas intervenciones mostraron efectos positivos sobre las variables individuales; sin embargo, resaltan la necesidad de realizar más estudios que comparen y evalúen la efectividad de cada uno de los componentes de dichos tratamientos, incluyendo programas complementarios enfocados en los ámbitos social y familiar que ofrezcan una intervención holística y efectiva (O'Callaghan et al., 2013).

En este sentido, las intervenciones familiares citadas en esta revisión sistemática (HF, SF, ADPAT, PMD) están diseñadas para mejorar y fortalecer las relaciones familiares y surgen en complementariedad con los tratamientos enfocados directamente en el niño o adolescente víctima. Los resultados encontrados en estas investigaciones en variables como la crianza positiva, la comunicación, la empatía y las habilidades sociales influyen directamente en el comportamiento y en las dificultades psicológicas del niño y el cuidador asociadas al evento traumático (Annan et al., 2017; De Voe et al., 2017; Gewirtz et al., 2018; Zhang et al., 2019), promoviendo de esta manera que los beneficios del tratamiento se constituyan de forma bidireccional, es decir, que todos los integrantes de la familia reciban y aporten a la generación de cambios positivos.

Las intervenciones sociales o escolares se basaron en estrategias dirigidas a los niños y adolescentes afectados por el trauma en contextos diferentes al clínico y familiar (ERASE, PAA, IBA, IPS, IDP, MAP, IPPE, IBD).
Las actividades grupales en un contexto de apoyo social y escolar resultaron efectivas para mejorar el bienestar psicológico de la población estudiada (Berger et al., 2012; Dajani et al., 2018; Influs et al., 2019; O'Callaghan et al., 2014; Panter-Brick et al., 2018; Richards et al., 2014; Shechtman y Mor, 2010; Slone et al., 2013). Aunque una de las intervenciones identificadas no encontró evidencias directas del tratamiento (IBA), los autores especifican la mediación de las variables individuales y sociales como la edad (menores de 11 años), el género femenino y la convivencia familiar con ambos padres como factores protectores en contextos de guerra (Jordans et al., 2010; Tol et al., 2014).

Variables como la edad, el género y el funcionamiento familiar hacen difícil la tarea de promover cambios significativos en cualquier tipo de población, especialmente en personas que han sido víctimas de conflictos (migración forzosa, abandono parental, carencia de necesidades básicas, ansiedad, estrés, miedo, ira, desesperanza y depresión, entre otras). No obstante, sobresale el interés y la existencia de programas de calidad enfocados en actividades deportivas que buscan la promoción de la salud mental y la generación de espacios de consolidación de paz (Richards et al., 2014). Además, se resalta que algunos estudios informan sobre la falta de eficacia de los tratamientos empleados, hecho que permite esclarecer el camino para investigaciones futuras, ya que futuros investigadores pueden tomar como referencia los hallazgos no favorables de estos estudios y replantear aspectos metodológicos que permitan cambios positivos en la población infanto-juvenil víctima de conflicto armado (Jordans et al., 2010; Panter-Brick et al., 2018; Puffer et al., 2015; Tol et al., 2014).

La mayoría de las intervenciones incluidas no contaban con el personal adecuado para desarrollar el tratamiento y, aunque los terapeutas recibían formación durante un tiempo determinado, no tenían un título profesional ni la experiencia necesaria para ejecutar este tipo de tareas. En varios casos, el personal implicado pertenecía a la comunidad debido a su relación directa con los niños y adolescentes víctimas o a razones de tiempo y coste económico (Annan et al., 2017; Barron et al., 2016; Catani et al., 2019; Dawson et al., 2018). A pesar de ello, algunas intervenciones basadas en TCC y empleadas en contextos educativos evidencian resultados prometedores con tamaños del efecto moderados principalmente sobre TEPT, depresión y ansiedad. Sin embargo, existe la necesidad de emplear estrategias poco exploradas hasta el momento como las comunitarias y familiares que también han presentado efectos positivos en problemas conductuales, emocionales y habilidades sociales (Gillies et al., 2017; Jordans et al., 2016; Purgato et al., 2018). 
Una limitación de esta revisión sistemática es que las intervenciones incluidas se centraban en distintos tipos de problemas psicopatológicos en función del tipo de estresor al que han sido expuestos los niños y adolescentes víctimas de conflicto armado como la migración forzosa, desintegración familiar, desaparición forzada u otros eventos traumáticos. Por esta razón, la comparación entre las intervenciones puede llevar a conclusiones parciales que no describen completamente la realidad de los participantes. Otra limitación es que todos los estudios encontrados estaban escritos en inglés y en bases de datos electrónicas, lo que excluye estudios potencialmente relevantes publicados en fuentes de información impresa así como literatura gris o en otros idiomas como el portugués. Por último, los términos clave se buscaron en los títulos y los resúmenes de los trabajos, excluyendo artículos que trataran el tema como componente secundario del mismo.

En conclusión, se destaca el interés creciente por la intervención en contextos de crisis humanitarias, no solo en el ámbito de la salud física sino también en los aspectos concernientes a la salud mental, que generalmente se han considerado en un segundo o tercer nivel de relevancia. Se destaca la existencia de ensayos clínicos de calidad con la inclusión de muestras representativas, evaluaciones de seguimiento y tratamientos eficaces que incluyen a la familia del niño como eje de posibles cambios positivos a nivel cognitivo, emocional y comportamental. A pesar de ello, se requiere la realización de un mayor número estudios que empleen metodologías apropiadas que permitan realizar múltiples mediciones y un seguimiento a largo plazo de variables que incluyan aspectos individuales, familiares y sociales (Gillies et al., 2017; Jordans et al., 2016; Villanueva-Bonilla y Ríos-Gallardo, 2018).

Las familias que han sido víctimas de conflicto armado y los profesionales que las han atendido podrán beneficiarse de los resultados y conclusiones descritas en la presente revisión, especialmente de cara a informarse sobre las intervenciones más eficaces para la población infanto-juvenil víctima de conflicto armado.

\section{Conflictos de intereses}

Los autores declaran no tener conflictos de intereses.

\section{Referencias $^{14}$}

*Annan, J., Sim, A., Puffer, E. S., Salhi, C. \& Betancourt, T. S. (2017). Improving mental health outcomes of burmese migrant

\footnotetext{
${ }^{14}$ Los artículos incluidos en la revisión sistemática están señalados con un asterisco.
}

and displaced children in thailand: A community-based randomized controlled trial of a parenting and family skills intervention. Prevention Science, 18(7), 793-803. https://doi. org/10.1007/s11121-016-0728-2

*Barron, I., Abdallah, G. \& Heltne, U. (2016). Randomized control trial of teaching recovery techniques in rural occupied palestine: Effect on adolescent dissociation. Journal of Aggression, Maltreatment and Trauma, 25(9), 955-973. https://doi.org/10.1 080/10926771.2016.1231149

* Barron, I., Abdallah, G. \& Smith, P. (2013). Randomized control trial of a CBT trauma recovery program in palestinian schools. Journal of Loss \& Trauma, 18(4), 306-321. https://doi.org/10. 1080/15325024.2012.688712

*Berger, R., Gelkopf, M. \& Heineberg, Y. (2012). A teacherdelivered intervention for adolescents exposed to ongoing and intense traumatic war-related stress: A quasi-randomized controlled study. Journal of Adolescent Health, 51(5), 453461. https://doi.org/10.1016/j.jadohealth.2012.02.011

Betancourt, T. S., Borisova, I., Williams, T. P., Meyers-Ohki, S. E., Rubin-Smith, J. E., Annan, J. \& Kohrt, B. A. (2013a). Research review: Psychosocial adjustment and mental health in former child soldiers-A systematic review of the literature and recommendations for future research. Journal of Child Psychology and Psychiatry and Allied Disciplines, 54(1), 1736. https://doi.org/10.1111/j.1469-7610.2012.02620.x

Betancourt, T. S., Meyers-Ohki, S. E., Charrow, A. P. \& Tol, W. A. (2013b). Interventions for children affected by war: An ecological perspective on psychosocial support and mental health care. Harvard Review of Psychiatry, 21(2), 70-91. https://doi.org/10.1097/HRP.0b013e318283bf8f

Brown, F. L., de Graaff, A. M., Annan, J. \& Betancourt, T.S. (2017). Annual Research Review: Breaking cycles of violence-a systematic review and common practice elements analysis of psychosocial interventions for children and youth affected by armed conflict. Journal of Child Psychology and Psychiatry and Allied Disciplines, 58(4), 507-524. https://doi.org/10.1111/ jcpp. 12671

Castro-Camacho, L., Moreno, J. D. \& Naismith, I. (2019). Contextual adaptation of the unified protocol for the transdiagnostic treatment of emotional disorders in victims of the armed conflict in Colombia: A case study. Cognitive and Behavioral Practice, 26(2), 366-380. https://doi.org/10.1016/j. cbpra.2018.09.002

*Catani, C., Kohiladevy, M., Ruf, M., Schauer, E., Elbert, T. \& Neuner, F. (2009). Treating children traumatized by war and Tsunami: A comparison between exposure therapy and meditation-relaxation in North-East Sri Lanka. BMC Psychiatry, 9-22. https://doi.org/10.1186/1471-244x-9-22

*Dajani, R., Hadfield, K., van Uum, S., Greff, M. \& Panter-Brick, C. (2018). Hair cortisol concentrations in war-affected adolescents: A prospective intervention trial. Psychoneuroendocrinology, 89, 138-146. https://doi.org/10.1016/j.psyneuen.2017.12.012

*Dawson, K., Joscelyne, A., Meijer, C., Steel, Z., Silove, D., \& Bryant, R.A. (2018). A controlled trial of trauma-focused therapy versus problem-solving in Islamic children affected by civil conflict and disaster in Aceh, Indonesia. Australian 
and New Zealand Journal of Psychiatry, 52(3), 253-261. https://doi.org/10.1177/0004867417714333

*De Voe, E. R., Paris, M. A. R., Ross, A. \& Acker, M. (2017). A randomized clinical trial of a postdeployment parenting intervention for service members and their families with very young children. Psychological Trauma: Theory, Research, Practice, and Policy, 9, 25-34. https://doi.org/10.1037/tra0000196

Deblinger, E., Mannarino, A. P., Cohen, J. A., Runyon, M. K. \& Steer, R. A. (2011). Trauma-focused cognitive behavioral therapy for children: Impact of the trauma narrative and treatment length. Depression and Anxiety, 28(1), 67-75. https://doi.org/10.1002/da.20744

Dimitry, L. (2012). A systematic review on the mental health of children and adolescents in areas of armed conflict in the Middle East. Child Care Health and Development, 38(2), 153-161. https://doi.org/10.1111/j.1365-2214.2011.01246.x

*Gewirtz, A. H., DeGarmo, D. S. \& Zamir, O. (2018). After deployment, adaptive parenting tools: 1-year outcomes of an evidence-based parenting program for military families following deployment. Prevention Science, 19(4), 589-599. https://doi.org/10.1007/s11121-017-0839-4

Gillies, D., Maiocchi, L., Bhandari, A. P., Taylor, F., Gray, C. \& O'Brien, L. (2016). Psychological therapies for children and adolescents exposed to trauma. Cochrane Database of Systematic Reviews, 10, 1-221. https://doi.org/10.1002/14651858.CD012371

Higgins, J. P. T. \& Green, S. (Eds.) (2011). Cochrane handbook for systematic reviews of interventions version 5.1.0 [updated March 2011]. The Cochrane Collaboration, 2011. www. cochrane-handbook.org.

*Influs, M., Masalha, S., Zagoory-Shaon, O. \& Feldman, R. (2019). Dialogue intervention to youth amidst intractable conflict attenuates stress response to outgroup. Hormones and Behavior, 110, 68-76. https://doi.org/10.1016/j.yhbeh.2019.02.013

Jordans, M., Pigott, H. \& Tol, W.A. (2016). Interventions for children affected by armed conflict: A systematic review of mental health and psychosocial support in low- and middleincome countries. Current Psychiatry Reports, 18(1), 1-15. https://doi.org/10.1007/s11920-015-0648-z

*Jordans, M. J., Komproe, I. H., Tol, W. A., Kohrt, B. A., Luitel, N. P., Macy, R. D. \& de Jong, J.T. (2010). Evaluation of a classroom-based psychosocial intervention in conflictaffected Nepal: A cluster randomized controlled trial. Journal of Child Psychology and Psychiatry, 51(7), 818-826. https:// doi.org/10.1111/j.1469-7610.2010.02209.x

Jordans, M J. D., Tol, W. A., Komproe, I. H. \& De Jong, J. V. T. M. (2009). Systematic review of evidence and treatment approaches: Psychosocial and mental health care for children in war. Child and Adolescent Mental Health, 14(1), 2-14. https://doi. org/10.1111/j.1475-3588.2008.00515.x

*Kalantari, M., Yule, W., Dyregrov, A., Neshatdoost, H. \& Ahmadi, S.J. (2012). Efficacy of writing for recovery on traumatic grief symptoms of afghani refugee bereaved adolescents: A randomized control trial. Omega (United States), 65(2), 139150. https://doi.org/10.2190/OM.65.2.d

*Lange-Nielsen, I. I., Kolltveit, S., Thabet, A. A. M., Dyregrov, A., Pallesen, S., Johnsen, T. B. \& Laberg, J. C. (2012). Shortterm effects of a writing intervention among adolescents in Gaza. Journal of Loss y Trauma, 17(5), 403-422. https://doi.or $\mathrm{g} / 10.1080 / 15325024.2011 .650128$
Layne, C. M., Saltzman, W. R., Poppleton, L., Burlingame, G. M., Pašalić, A., Duraković, E., Music, M., Campara, N., Dapo, N., Arslanagic, B., Steinberg, A. \& Pynoos, R. S. (2008). Effectiveness of a school-based group psychotherapy program for war-exposed adolescents: A randomized controlled trial. Journal of the American Academy of Child and Adolescent Psychiatry, 47(9), 1048-1062. https://doi. org/10.1097/CHI.0b013e31817eecae

Lee-Koo, K. (2018). 'The intolerable impact of armed conflict on children': The united nations security council and the protection of children in armed conflict. Global Responsibility to Protect, 10(1-2), 57-74. https://doi.org/10.1163/1875984X-01001004

Márquez, Y. I., Deblinger, E. \& Dovi, A. T. (2020). The value of trauma-focused cognitive behavioral therapy (TF-CBT) in addressing the therapeutic needs of trafficked youth: A case study. Cognitive and Behavioral Practice, 1-57. https://doi. org/10.1016/j.cbpra.2019.10.001

*McMullen, J., O'Callaghan, P., Shannon, C., Black, A. \& Eakin, J. (2013). Group trauma-focused cognitive-behavioural therapy with former child soldiers and other war-affected boys in the DR Congo: A randomised controlled trial. Journal of Child Psychology \& Psychiatry, 54(11), 1231-1241. https:// doi.org/10.1111/jcpp. 12094

Moher, D., Liberati, A., Tetzlaff, J. \& Altman, D. G. (2009). Preferred reporting items for systematic reviews and metaanalyses: The PRISMA statement. Annals of internal medicine, 151(4), 264-269.

*Murray, L. K., Skavenski, S., Kane, J. C., Mayeya, J., Dorsey, S., Cohen, J. A., Michalopoulos, L., Imasiku, M. \& Bolton, P. A. (2015). Effectiveness of trauma-focused cognitive behavioral therapy among trauma-affected children in Lusaka, Zambia A randomized clinical trial. Jama Pediatrics, 169(8), 761-769. https://doi.org/10.1001/jamapediatrics.2015.0580

Norwegian Refugee Council. (2019). Global report on internal displacement 2019. https://www.internal-displacement.org/ global-report/grid2019/

*O'Callaghan, P., Branham, L., Shannon, C., Betancourt, T. S., Dempster, M. \& McMullen, J. (2014). A pilot study of a family focused, psychosocial intervention with war-exposed youth at risk of attack and abduction in north-eastern Democratic Republic of Congo. Child Abuse and Neglect, 38(7), 11971207. https://doi.org/10.1016/j.chiabu.2014.02.004

*O'Callaghan, P., McMullen, J., Shannon, C., Rafferty, H. \& Black, A. (2013). A randomized controlled trial of traumafocused cognitive behavioral therapy for sexually exploited, war-affected congolese girls. Journal of the American Academy of Child y Adolescent Psychiatry, 52(4), 359-369. https://doi.org/10.1016/j.jaac.2013.01.013

*Ooi, C. S., Rooney, R. M., Roberts, C., Kane, R. T., Wright, B. \& Chatzisarantis, N. (2016). The Efficacy of a group cognitive behavioral therapy for war-affected young migrants living in Australia: A cluster randomized controlled trial. Frontiers in Psychology, 7, 1-14. https://doi.org/10.3389/fpsyg.2016.01641

Oprel, D. A. C., Hoeboer, C. M., Schoorl, M., De Kleine, R. A., Wigard, I. G., Cloitre, M., Van Minnen, A. \& Van Der Does, W. (2018). Improving treatment for patients with childhood abuse related posttraumatic stress disorder (IMPACT study): Protocol for a multicenter randomized trial comparing prolonged exposure with intensified prolonged exposure and phase-based 
treatment. BMC Psychiatry, 18(1), 1-10.https://doi.org/10.1186/ s12888-018-1967-5

*Panter-Brick, C., Dajani, R., Eggerman, M., Hermosilla, S., Sancilio, A. \& Ager, A. (2018). Insecurity, distress and mental health: Experimental and randomized controlled trials of a psychosocial intervention for youth affected by the Syrian crisis. Journal of Child Psychology and Psychiatry and Allied Disciplines, 59(5), 523-541. https://doi.org/10.1111/jcpp.12832

*Puffer, E. S., Green, E. P., Chase, R. M., Sim, A. L., Zayzay, J., Friis, E., García-Rolland, E. \& Boone, L. (2015). Parents make the difference: A randomized-controlled trial of a parenting intervention in Liberia. Global Mental Health, 2, 1-13. https://doi.org/10.1017/gmh.2015.12

Purgato, M., Gastaldon, C., Papola, D., van Ommeren, M., Barbui, C. \& Tol, W.A. (2018). Psychological therapies for the treatment of mental disorders in low- and middle-income countries affected by humanitarian crises. Cochrane Database of Systematic Reviews, 6, 390-400. https://doi.org/10.1002/14651858.CD011849.pub2

*Qouta, S. R., Palosaari, E., Diab, M. \& Punamaki, R.-L. (2012). Intervention effectiveness among war-affected children: A cluster randomized controlled trial on improving mental health. Journal of Traumatic Stress, 25(3), 288-298. https:// doi.org/10.1002/jts. 21707

*Richards, J., Foster, C., Townsend, N. \& Bauman, A. (2014). Physical fitness and mental health impact of a sport-fordevelopment intervention in a post-conflict setting: randomised controlled trial nested within an observational study of adolescents in Gulu, Uganda. BMC Public Health, 14, 1-13. https://doi.org/10.1186/1471-2458-14-619

*Robjant, K., Koebach, A., Schmitt, S., Chibashimba, A., Carleial, S. \& Elbert, T. (2019). The treatment of posttraumatic stress symptoms and aggression in female former child soldiers using adapted narrative exposure therapy - a RCT in Eastern Democratic Republic of Congo. Behaviour Research and Therapy, 123, 1-10. https://doi.org/10.1016/j.brat.2019.103482

*Shechtman, Z. \& Mor, M. (2010). Groups for children and adolescents with trauma-related symptoms: Outcomes and processes. International Journal of Group Psychotherapy, 60(2), 221-244. https://doi.org/10.1521/ijgp.2010.60.2.221

*Slone, M., Shoshani, A. \& Lobel, T. (2013). Helping youth immediately following war exposure: A randomized controlled trial of a school-based intervention program. Journal of Primary Prevention, 34(5), 293-307. https://doi.org/10.1007/ s10935-013-0314-3

*Tol, W.A., Komproe, I. H., Jordans, M. J., Ndayisaba, A., Ntamutumba, P., Sipsma, H., Smallegange, E., Macy, R. \& de Jong, J. T. (2014). School-based mental health intervention for children in war-affected Burundi: A cluster randomized trial. BMC Med, 12, 1-12. https://doi.org/10.1186/1741-7015-12-56

Tol, W. A., Song, S. \& Jordans, M. J. D. (2013). Annual research review: Resilience and mental health in children and adolescents living in areas of armed conflict - A systematic review of findings in low- and middle-income countries. Journal of Child Psychology and Psychiatry and Allied Disciplines, 54(4), 445460. https://doi.org/10.1111/jcpp.12053

Villanueva-Bonilla, C. y Ríos-Gallardo, Á. M. (2018). Factores protectores y de riesgo del trastorno de conducta y del trastorno de déficit de atención e hiperactividad. Una revisión sistemática. Revista de Psicopatología y Psicología Clínica, 23(1), 59-73.

*Zhang, N., Zhang, J. \& Gewirtz, A. H. (2019). Do less mindful mothers show better parenting via improvements in trait mindfulness following a military parent training program? Frontiers in Psychology, 10, 1-14. https://doi.org/10.3389/ fpsyg.2019.00909 